\title{
25 Research Square \\ No Or Diffuse Phase-Transition With Temperature In One-Dimensional Ising Model?
}

\section{Li Zhang}

Nanjing University

\section{Li-Li Zhang}

Yili Normal University

Yi-Neng Huang ( $\sim$ ynhuang@nju.edu.cn )

Nanjing University

\section{Research Article}

Keywords: Ising, one-dimensional, temperature, storage

Posted Date: October 18th, 2021

DOI: https://doi.org/10.21203/rs.3.rs-516637/v2

License: (1) This work is licensed under a Creative Commons Attribution 4.0 International License.

Read Full License 


\section{No or diffuse phase-transition with temperature in one-dimensional Ising model?}

Li Zhang ${ }^{1,2}$, Li-Li Zhang², and Yi-Neng Huang ${ }^{2,1^{*}}$

${ }^{1}$ National Laboratory of Solid State Microstructures, School of Physics, Nanjing University, China.

${ }^{2}$ Xinjiang Laboratory of Phase Transitions and Microstructures in Condensed Matters, College of Physical Science and Technology, Yili Normal University, China.

*Corresponding author. Email: ynhuang@nju.edu.cn

\section{Abstract}

For about a century since Ising model, one of the most important microscopic models of physics, was proposed, it is agreed that there is no phase-transition with temperature in the onedimensional based on no global spontaneous-magnetization. On the contrary, our exact calculation of all-size spontaneous-magnetization and computer simulations of spin orientation states show that the low temperature state of one-dimensional Ising model (1D-IM) is a ferromagnetic phase of multi-domains, and it is concluded that the transition process from high temperature paramagnetic to low temperature ferromagnetic phase is a diffuse phase-transition by comparing with the corresponding phenomena. Moreover, based on the previous outcome that $1 \mathrm{D}-\mathrm{IM}$ is always at paramagnetic state in whole temperature range, the corresponding onedimensional materials cannot be used in storage and memory in principle. However, our results of the low temperature ferromagnetic state in 1D-IM indicate that the relevant materials have potential applications in storage devices.

\section{INTRODUCTION}

In the nearly 100 years since Ising model (IM) ${ }^{1,2}$, one of the most important microscopic models of physics ${ }^{1}$, especially in the field of phase-transition ${ }^{1,3-8}$, was proposed in 1920 , it is agreed that there is no temperature dependent phase-transition in the one-dimensional. This is because the calculated global spontaneous-magnetization of the model system is zero in whole temperature range $\mathrm{e}^{2,3}$.

It should be pointed out that the absence of global spontaneous-magnetization does not deny the possible existence of spontaneous-magnetization regions of finite-sizes, such as multidomain ferromagnetic state ${ }^{9,10}$, in 1D-IM. In order to solve this problem, we need to get the allsize spontaneous-magnetization of this model, but to the best of the authors' knowledge, there has been no research on it so far.

Moreover, 1D-IM has a diffuse heat-capacity (specific-heat) peak around a certain temperature ${ }^{2,3}$. As an analogy, there is also a diffuse heat-capacity peak with temperature in the heterogeneous systems, and this peak reflects the diffuse phase-transition ${ }^{11-13}$. Corresponding to this transition, the order-parameter ${ }^{14-17}$ is also spatially inhomogeneous ${ }^{18,19}$, i.e. it can mainly be described by the finite-size part of all-size order-parameter ${ }^{20}$.

In this paper, the all-size spontaneous-magnetization, i.e. spontaneous magnetization in all sizes (Eq.3), with temperature in 1D-IM is calculated accurately, and the results show that the low temperature state of this model is a ferromagnetic phase of multi-domains ${ }^{9,10,21-23}$. Our computer simulation of spin orientation state ${ }^{24,25}$ also confirm this conclusion intuitively. Moreover, it is concluded that the transition process from high temperature paramagnetic to low temperature ferromagnetic phase is a diffuse phase-transition by comparing with the corresponding phenomena ${ }^{14-19,26-29}$. 
The Hamiltonian $\left(H_{1 \mathrm{D}-\mathrm{IM}}\right)$ of $1 \mathrm{D}-\mathrm{IM}^{2,3}$ is,

$$
H_{1 \mathrm{D}-\mathrm{IM}}=\lim _{N \rightarrow \infty}\left[-J \sum_{i=1}^{N-1} \sigma_{i} \sigma_{i+1}\right]
$$

in which $\sigma_{i}$ is the ith spin and $\sigma_{i}= \pm 1, J$ the interaction energy constant between the nearestneighbor spins, and $N$ the total number of spins in the model system.

In the model, the magnetic-moment $\left(s_{l}^{r}\right)$ including arbitrary $l$ nearest-neighbor spins or spin segment with length $l$ spins is,

$$
s_{l}^{r} \equiv \mu \sum_{i=0}^{l-1} \sigma_{r+i}
$$

where $\mu$ is the magnetic moment of a spin, and $r$ expresses an arbitrary reference site.

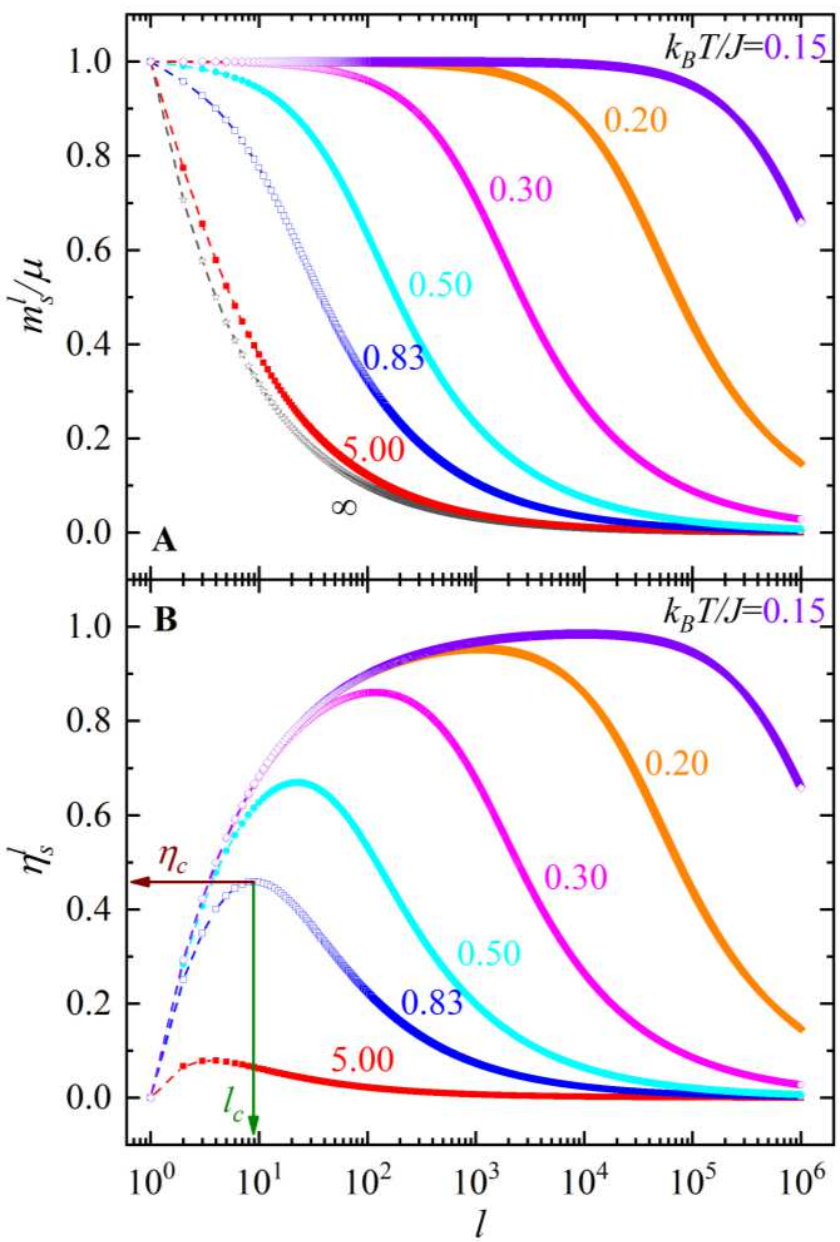

Fig. 1 For series temperature $(T),(\mathrm{A})$ all-size spontaneous-magnetization $\left(m_{s}^{l}\right)$ and (B) all-size order-parameter $\left(\eta_{s}^{l}\right)$ vs the spin number $(l)$ of spin segments in one-dimensional Ising model.

To describe the temperature dependence of the amplitude of $s_{l}^{r}$ (excluding the orientations corresponding to its signs) in this paper, the all-size spontaneous-magnetization $\left(m_{s}^{l}\right)$ of $1 \mathrm{D}$ IM is defined as (see METHODS), 


$$
\begin{aligned}
m_{s}^{l} & \equiv \frac{1}{l} \sqrt{\lim _{n \rightarrow \infty} \frac{1}{Z_{N}} \sum_{\sigma_{i}= \pm 1, \cdots \sigma_{n}= \pm 1}\left(s_{l}^{r}\right)^{2} \exp \left[\frac{J}{k_{B} T} \sum_{i=1}^{N-1} \sigma_{i} \sigma_{i+1}\right]} \\
& =\frac{\mu}{l} \sqrt{2\left[\frac{l-\gamma^{l}}{1-\gamma}-\frac{\gamma\left(1-\gamma^{l-1}\right)}{(1-\gamma)^{2}}\right]-l}
\end{aligned}
$$

here $k_{B}$ is Boltzmann constant, $T$ the temperature of the heat bath in which the onedimensional spin chain is located, $Z_{N}$ the partition function of the spin orientation ensemble of 1D-IM, and $\gamma \equiv \tanh \left(\frac{J}{k_{B} T}\right)$.

Obviously, $m_{s}^{\infty}$ is the global spontaneous-magnetization when $l \rightarrow \infty$. From Eq. 3, we can get that $m_{S}^{\infty}=0$ except $T \rightarrow 0$, which is consistent with the past result ${ }^{2,3}$.

Fig. 1A shows $m_{s}^{l}$ vs $l$ for series $T$, and it can be seen that: (i) At high temperature (e.g. $\left.T=5.00 \mathrm{~J} / k_{B}\right), m_{s}^{l}$ decreases rapidly with increasing $l$, which indicates that the spatial scale of spontaneous-magnetization is small; and (ii) At low temperature (e.g. $\left.T=0.15 \mathrm{~J} / k_{B}\right), m_{s}^{l} \rightarrow$ $\mu$ in a large range of $l$, which states clearly that the spontaneous-magnetization regions not only have a large size, but also almost all the spins in the regions point along the same orientation.

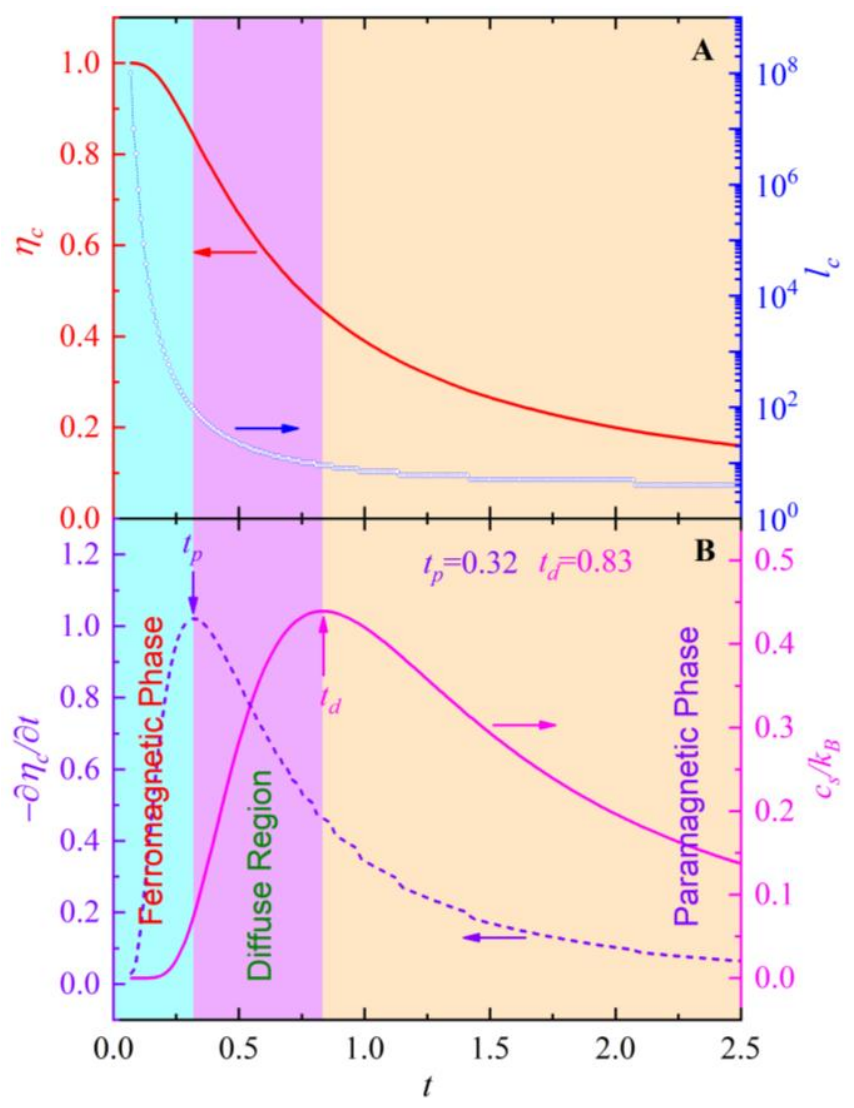

Fig. 2 (A) characteristic spontaneous-magnetization $\left(\eta_{c}\right)$ and its characteristic-size $\left(l_{c}\right)$, as well as (B) $-\partial \eta_{c} / \partial t$ and heat capacity per spin $\left(c_{S}\right)$ in 1D-IM vs reduced temperature $\left(t \equiv k_{B} T / J\right)$.

\section{DISCUSSION}

According to Landau theory ${ }^{30}$, the order-parameter is the key of phase-transition, which characterizes the relative change of low to high temperature phase. It should be noted that the 
order-parameter of Landau theory is essentially global order-parameter, which is defined on the size of micro-infinitely-great and macro-infinitely-small. Here, all-size order-parameter $\left(\eta_{s}^{l}\right)$ is introduced, that is the relative variation of $m_{s}^{l}$ to its high temperature limit value $\left[m_{s}^{l}(T \rightarrow \infty)\right]$,

$$
\eta_{s}^{l} \equiv \frac{m_{s}^{l}-m_{s}^{l}(T \rightarrow \infty)}{\mu}
$$

In fact, $m_{s}^{l}(T \rightarrow \infty)$ represents the autocorrelation $\left(\sigma_{i}{ }^{2}\right)$ of a spin (Eq. 3 and M5 in METHODS), and of course has nothing to do with the phase-transition process.
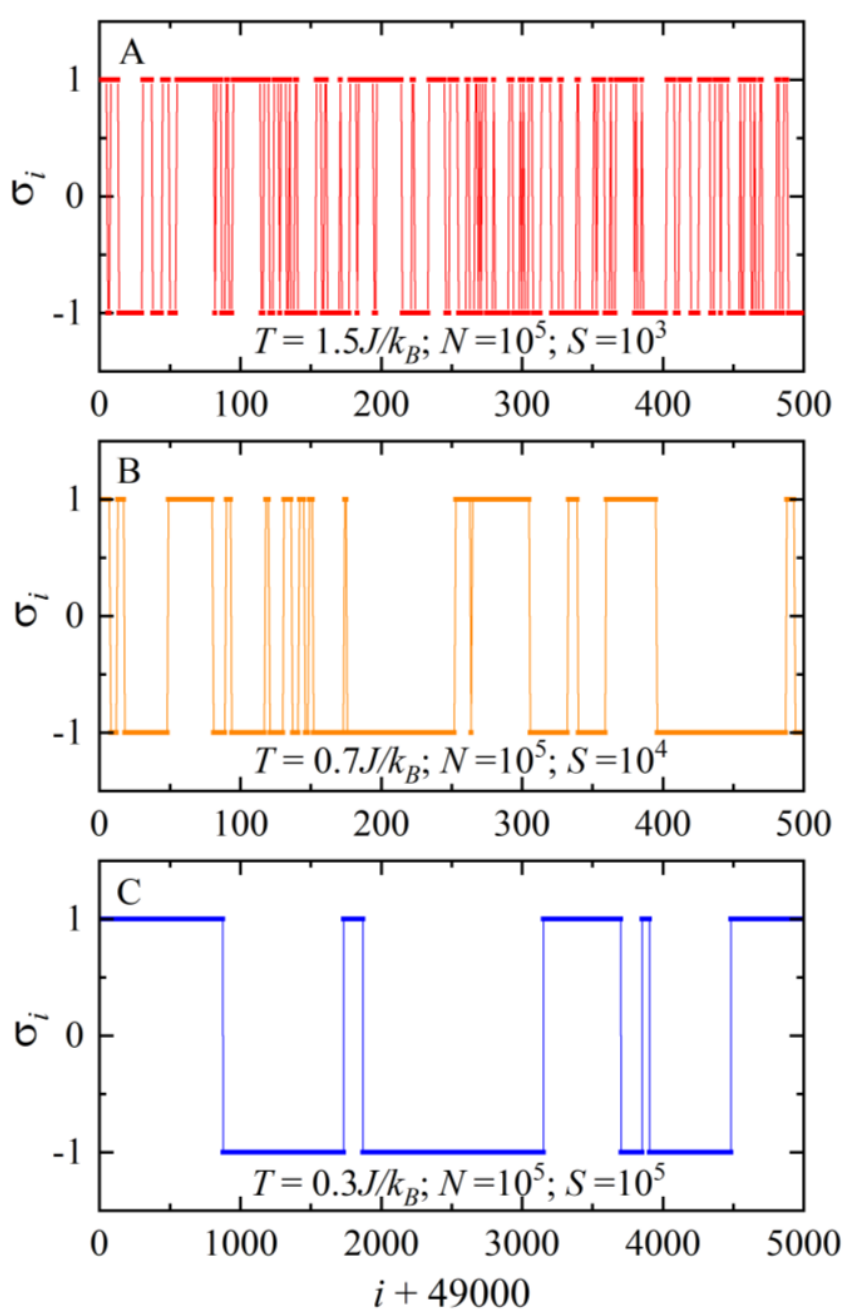

Fig. 3 For $T=1.5 \mathrm{~J} / k_{B}(\mathrm{~A}), 0.7 \mathrm{~J} / k_{B}(\mathrm{~B})$, and $0.3 \mathrm{~J} / k_{B}(\mathrm{C})$, spin orientation states $\left(\sigma_{i}\right)$ vs their sites $(i)$ in the segments [500 (A), $500(B)$, and $5000(C)]$ from the reference position (49000) of one-dimensional Ising model with chain length $\left(N=10^{5}\right)$ at thermal equilibrium after certain Monte Carlo steps $(S)$.

At series $T, \eta_{s}^{l}$ vs $l$ is shown in Fig. 1B, which indicates that for all $T, \eta_{s}^{l}$ has a single diffuse peak as a function of $l$. In this paper, the maximum value of $\eta_{s}^{l}$ is expressed as $\eta_{c}$, and the corresponding value of $l$ as $l_{c}$. Obviously, $\eta_{c}$ and $l_{c}$ can be used as the characteristic parameters to describe the intensity and size of all-size spontaneous-magnetization, so here $\eta_{c}$ is called the characteristic-order-parameter and $l_{c}$ the characteristic-size of $\eta_{c}$ in 1D-IM. Moreover, the dispersion of the $\eta_{s}^{l}$ peak shows that both the size of the spontaneousmagnetization regions and its internal magnetization have obvious distribution. 
$\eta_{c}$ and $l_{c}$ vs $T$ (Fig. 2A) show that, with decreasing $T$ : (a) $\eta_{c}$ first increases slowly, then rapidly, and slowly again; and (b) $l_{c}$ first increases slowly and then rapidly. As an analogy, the simulated spin orientation states $\left(\sigma_{i}\right)$ vs their sites $(i)$ in the segments from the reference position of a spin chain at thermal equilibrium for different $T$ is plotted in Fig. 3 (see Methods) ${ }^{24,25}$, which shows that: (a) When $T$ is high, the regions with the same $\sigma_{i}$ value is small (Fig. 3A for $T=1.5 \mathrm{~J} / k_{B}$ ); (b) With decreasing $T$, the average scale of such regions increases rapidly (Fig. 3B for $T=0.7 J / k_{B}$ ); and (c) For $T=0.3 J / k_{B}$, the average size has reached $\sim 10^{3}$ spins (Fig. 3C), and it can be imagined that the size will be larger if $T$ is lower.

The low temperature state of $1 \mathrm{D}-\mathrm{IM}$ is first discussed here. For $T=0.07 \mathrm{~J} / k_{B}, \eta_{c}=$ 0.9999 and $l_{c}=10^{8}$. If the lattice parameter of $1 \mathrm{D}-\mathrm{IM}$ is assumed to be $0.3 \mathrm{~nm}$, the characteristic-size of the spontaneous-magnetization regions will reach macroscopic $3 \mathrm{~cm}$. When $T<0.07 J / k_{B}, \eta_{c}$ and $l_{c}$ will become larger, as shown in Fig. 2A. The computer simulation results also intuitively confirm this conclusion (Fig. 3C). Such macroscopic scale regions of almost completely spontaneous-magnetization show that 1D-IM has ferromagneticregions or domains ${ }^{9,10,21-23}$ of macroscopic scale at non-zero low temperature. In addition, $\eta_{s}^{l} \rightarrow$ 0 when $l \rightarrow \infty$, i.e. $\eta_{s}^{\infty} \rightarrow 0$ (Fig. 1B and Eqs. 3-4), indicating that $1 \mathrm{D}-\mathrm{IM}$ is at the ferromagnetic multi-domain state ${ }^{9,10,21-23}$ at low temperature.

According to the definition of ferromagnetic phase ${ }^{9,10}$, i.e. there is non-zero or measurable uniform spontaneous-magnetization in a size much larger than the lattice constant, and by comparing with corresponding experimental results ${ }^{18,19,21-23}$, it can be concluded that the state of 1D-IM at non-zero low temperature is a ferromagnetic phase.

By analogy: (a) The domain size in the ferromagnetic and ferroelectric phases of traditional ferromagnetic and ferroelectric single crystals at room temperature is about $\mu \mathrm{m}$ to $\mathrm{mm}^{21-23}$, such as $\sim 2 \times 10^{-2} \mathrm{~mm}, \sim 1 \mathrm{~mm}$ and $\sim 2 \mathrm{~mm}$ for Si-Fe ${ }^{21}, \mathrm{TGS}^{22}$ and $\mathrm{LiNbO}_{3}{ }^{23}$, respectively; and (b) The domain size in the ferroelectric phase of the relaxor-ferroelectric single crystal at room temperature is about $1 \mu \mathrm{m}^{18,19}$, for example, $\sim 1 \mu \mathrm{m}$ for both $\mathrm{PbMgNbO}_{3}$ and $\mathrm{Sr} 0.61 \mathrm{Ba} 0.31 \mathrm{NbO}_{6}{ }^{19}$.

Since 1D-IM must be of paramagnetic phase when $T$ is high enough, some transition process from paramagnetic to ferromagnetic phase must occur during cooling, and the process is diffuse with temperature (Fig. 2A).

It is worth noting that, traditionally, the phase-transition classification of Ehrenfest ${ }^{31}$ is based on the specific singularities of the thermodynamic potential, although this cannot be directly verified experimentally. The exact solutions of the heat-capacity and global spontaneous-magnetization of 2D-Ising model ${ }^{3}$ given by Onsager ${ }^{32}$ and Yang $^{33}$, respectively, do confirm this classification theoretically. However, it is gradually found that there is diffuse phase-transition without the singularities of the thermodynamic potential in the componentheterogeneous ${ }^{14-17,26,27}$ and finite-size ${ }^{28,29}$ systems with time.

By comparing the order-parameters ${ }^{14-17,26}$, heat-capacity ${ }^{11-13}$, and domain structure evolution ${ }^{18,19}$ of existing diffuse phase-transition with the diffuse variation of $\eta_{c}$ (Fig. 2A), the diffuse peak ${ }^{2,3}$ of heat-capacity $\left(c_{s}\right)$ per spin (Fig. 2B), and the diffuse transition between the nanoscale regions and macroscopic domains of spontaneous-magnetization ${ }^{9,10,21-23}$ as a function of $T$ (Fig. 1B and 2A), it can be concluded that a diffuse phase-transition between paramagnetic and ferromagnetic phases with temperature occurs in 1D-IM. Moreover, the diffuse heat-capacity peak ${ }^{2,3}$ with temperature is just a sign of the diffuse phase-transition in this model.

Moreover, several one-dimensional Ising and Heisenberg models with decorated structures, have shown pseudo-transitions with temperature ${ }^{34-36}$. Specifically, the first 
derivatives of the thermodynamic potential, such as entropy, internal energy, and spontaneousmagnetization, show a significant jump as a function of temperature, maintaining a close similarity with $1^{\text {st }}$-order phase-transition. Similarly, the $2^{\text {nd }}$-order derivatives of potential thermodynamics, such as specific heat and magnetic susceptibility, resemble a typical $2^{\text {nd }}$-order phase-transition at finite temperature. The authors think that this may be due to the size increase of the original short-size order-parameter in such one-dimensional models under the influence of the decorated structures.

According to the method of Ref. ${ }^{20}$, the temperature corresponding to the maximum of $-\frac{\partial \eta_{c}}{\partial T}$ is defined as the characteristic temperature $\left(T_{p}\right)$ of diffuse phase-transition in 1D-IM, and it is obtained $T_{p}=0.32 \mathrm{~J} / k_{B}$ (Fig. 2B).

It should be pointed out that $T_{p}$ is lower than the peak temperature $\left(T_{d}=0.83 \mathrm{~J} / k_{B}\right)$ of $c_{s}$ (Fig. 2B). For $2^{\text {nd }}$-order or continuous phase-transition, the peak temperatures of heat-capacity and the negative of the derivative of order-parameter to $T$ are equal to each other ${ }^{30,32,33}$, thus the authors think that the difference between $T_{p}$ and $T_{d}$ just reflects the dispersion of the diffuse phase-transition. To describe this dispersion, the diffuse-degree $(\varphi)$ of the transition is defined as,

$$
\varphi \equiv \frac{T_{d}-T_{p}}{T_{d}+T_{p}}=0.44
$$

It is worth noting that the static susceptibility $\left(\chi_{s}\right)$ per spin in 1D-IM (Fig. 4) always increases rapidly with decreasing $T^{2,3}$, instead of the $\lambda$-type peak of $2^{\text {nd }}$-order phase-transition ${ }^{30}$, which is thought to be one of the key evidences that no phase-transition with temperature exist in this model.

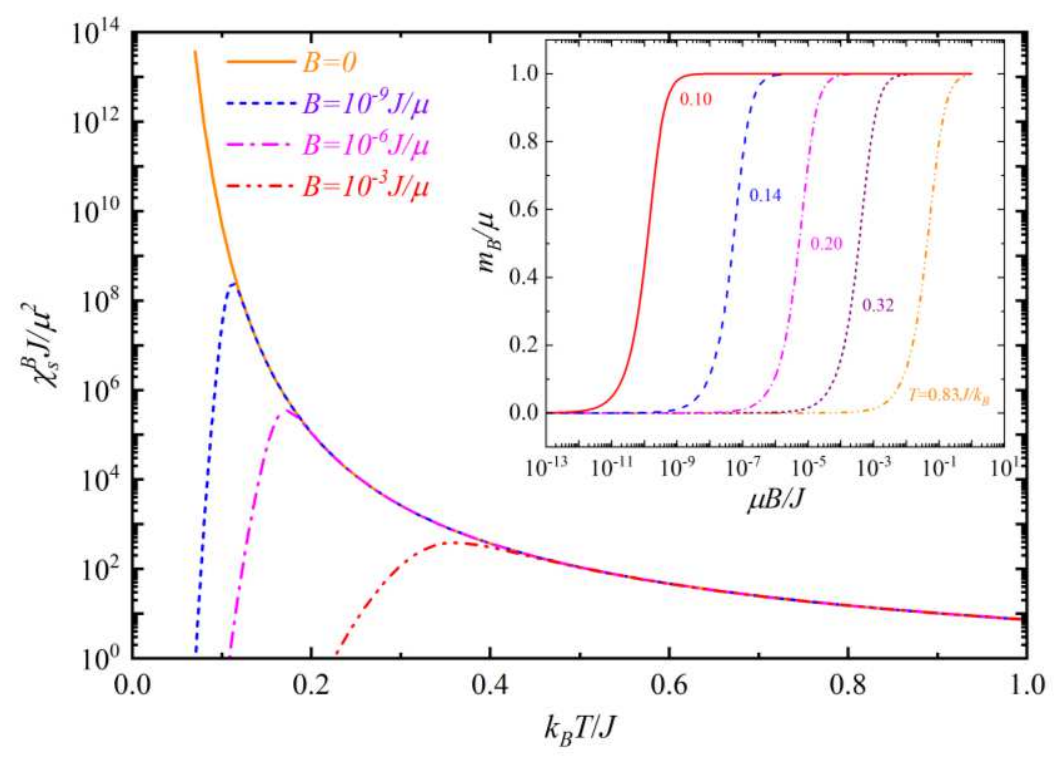

Fig. 4 Static susceptibility $\left(\chi_{s}^{B}\right)$ per spin vs $t$ for a series of external magnetic fields $(B)$ in onedimensional Ising model. The inset shows the magnetization $\left(m_{B}\right)$ per spin vs $B$ at series temperature $(T)$.

In order to further explore the micro-mechanism of the above characteristic of $\chi_{s}$, the static susceptibility $\left(\chi_{s}^{B}\right)$ per spin of 1D-IM in a fixed external magnetic field $(B)$ is calculated according to Ref. ${ }^{3}$, i.e. the magnetization $\left(m_{B}\right)$ per spin is, 
and $\chi_{s}^{B}$ is,

$$
m_{B}=\frac{\mu \sinh \left(\frac{\mu B}{k_{B} T}\right)}{\left[\sinh ^{2}\left(\frac{\mu B}{k_{B} T}\right)+\exp \left(-\frac{4 J}{k_{B} T}\right)\right]^{1 / 2}}
$$

here $b \equiv \mu B / J$ is reduced magnetic field.

$$
\chi_{s}^{B} \equiv \frac{\partial m_{B}}{\partial B}=\frac{\mu^{2}}{J} \frac{1}{t} \frac{\cosh \left(\frac{b}{t}\right) \exp \left(-\frac{4}{t}\right)}{\left[\sinh ^{2}\left(\frac{b}{t}\right)+\exp \left(-\frac{4}{t}\right)\right]^{3 / 2}}
$$

As shown in Fig. 4, for finite small $B$, there is a single diffuse peak of $\chi_{s}^{B}$ with $T$, and the peak temperature moves to high temperature with the increase of $B$. This is due to that a very small $B$ can saturate the magnetization of $1 \mathrm{D}-\mathrm{IM}$ at low temperature, e.g. the saturation $B \sim 10^{-9} \mathrm{~J} / \mu$ for $T=0.10 \mathrm{~J} / k_{B}$ (inset of Fig. 4 ), while the saturated magnetization leads to a smaller value of $\chi_{s}^{B}$. Because the saturation magnetization corresponds to the single domain state of the model, the increase of $\chi_{s}$ at low temperature is caused by the movement of domain walls $^{37-40}$.

In particular, because the measurement magnetic field used in experiments is always finite, the susceptibility peak (Fig. 4) will appear in 1D-IM as long as the experimental measurement is carried out. In other words, the continuously increasing characteristic of the theoretically predicted $\chi_{s}\left(\chi_{s}^{B=0}\right)$ with decreasing $T$ cannot be measured directly, which is only ideal value.

It should be noted that, when there is no external $B$, the magnetization of IM in thermal equilibrium must be zero at all temperature ${ }^{3,33}$. Therefore, the traditional method for calculating spontaneous-magnetization of this model is to first add a non-zero $B$ to IM and then calculate the magnetization of the model system. The magnetization obtained for $B \rightarrow 0$ is the spontaneous-magnetization of $\mathrm{IM}^{3}$, such as Yang's calculation of $2 \mathrm{D}-\mathrm{IM}^{33}$. This means that the low temperature ferromagnetic phase of $2 \mathrm{D}$-IM is a multi-domain state, and its coercive magnetic field tends to zero ${ }^{9,10}$. In other words, the above traditional method is not applicable to the model systems in which the coercivity field is not zero. As shown in the inset of Fig. 4, 1D-IM belongs to this class, and specifically, its coercive field is very small but does not tend to zero at low temperature except $T \rightarrow 0$. Obviously, the method for calculating all-size spontaneous-magnetization proposed in this paper does not have this problem, and the size of the magnetization regions can be obtained.

Moreover, according to the above traditional method, the calculated spontaneousmagnetization of finite-size 2D-IM is also zero in the whole temperature region, i.e. there is no phase-transition $^{3}$, although a diffuse heat-capacity peak with temperature appears in this model $^{41,42}$. On the contrary, it is generally believed that this model has phase-transition based the computer simulation results ${ }^{24,43,44}$. The authors think that a feasible way to solve this dispute is to calculate the all-size spontaneous-magnetization of the model, which needs further researches.

Although diffuse phase-transition is common in component-heterogeneous ${ }^{14-17,26,27,45-47}$ and small-size $28,29,48$ systems, there is no accurate calculation of the corresponding theoretical models so arr $^{20}$. Therefore, the exact results of the all-size order-parameter (Eq.4) in this paper may give some clues to the studies of this kind of phase-transition.

Traditionally, the description to $1^{\text {st }}$ and $2^{\text {nd }}$-order phase-transitions is based on global order-parameter (such as global spontaneous-magnetization and polarization etc.) ${ }^{2,3,9,10,30}$, but it cannot describe the spatially heterogeneous behavior of the order-parameter corresponding to the diffuse phase-transition in component-heterogeneous ${ }^{14-17,26,27,45-47}$ and small-size $28,29,48$ 
systems. Obviously, for the all-size order-parameter proposed in Ref. ${ }^{20}$ and this paper contains the global order-parameter, it can provide a unified description of $1^{\text {st }}$ and $2^{\text {nd }}$-order as well as diffuse phase-transitions.

Based on the previous conclusion that 1D-IM is always at paramagnetic state in whole temperature range $\mathrm{e}^{2,3}$, the corresponding one-dimensional materials cannot be used in storage and memory in principle. However, our results of the low temperature ferromagnetic state in 1D-IM (Fig. 2A) indicates that the relevant materials have potential applications in storage devices ${ }^{49,50}$. Therefore, this is also a problem worthy of in-depth study.

\section{METHODS}

Calculation of all-size spontaneous-magnetization

The method to calculate the all-size spontaneous-magnetization $\left(m_{s}^{l}\right)$ in 1D-IM is as the follows.

The partition function $\left(Z_{N}\right)$ of the spin orientation ensemble corresponding to $H_{1 \mathrm{D}-\mathrm{IM}}$ (Eq. 1) is,

$$
Z_{N} \equiv \sum_{\sigma_{1}= \pm 1, \cdots \sigma_{N}= \pm 1} \exp \left[v \sum_{i=1}^{N-1} \sigma_{i}^{1} \sigma_{i+1}^{1}\right]=2^{N} \cosh ^{N-1}(v)
$$

where $v \equiv \frac{J}{k_{B} T}$.

Let,

and according to,

$$
X_{l} \equiv \lim _{N \rightarrow \infty} \frac{1}{Z_{N}} \sum_{\sigma_{1}= \pm 1, \cdots \sigma_{N}= \pm 1}\left(s_{l}^{r}\right)^{2} \exp \left[v \sum_{i=1}^{N-1} \sigma_{i} \sigma_{i+1}\right]
$$

we obtain,

$$
\left(s_{l}^{r}\right)^{2}=\mu^{2}\left\{l+2\left[\sum_{i=1}^{l-1} \sigma_{r+i-1}^{1} \sigma_{r+i}^{1}+\sum_{i=2}^{l-1} \sigma_{r+i-2}^{1} \sigma_{r+i}^{1}+\cdots \sum_{i=l-1}^{l-1} \sigma_{r}^{1} \sigma_{r+l-1}^{1}\right]\right\}
$$

$$
X_{l}=\mu^{2}\left[l+2 \sum_{k=1}^{l-1}(l-k) \zeta_{k}\right]
$$

where $\zeta_{k}$ is the correlation function between $\sigma_{r}$ and $\sigma_{r+k}$, i.e.

$$
\zeta_{k} \equiv \lim _{N \rightarrow \infty} \frac{1}{Z_{N}} \sum_{\sigma_{1}= \pm 1, \cdots \sigma_{N}= \pm 1} \sigma_{r} \sigma_{r+k} \exp \left[v \sum_{i=1}^{N-1} \sigma_{i} \sigma_{i+1}\right]
$$

$$
=\lim _{N \rightarrow \infty} \frac{1}{2^{N}} \sum_{\sigma_{1}= \pm 1, \cdots \sigma_{N}= \pm 1} \prod_{i=1}^{N-1}\left(1+\gamma \sigma_{i} \sigma_{i+1}\right) \sigma_{r} \sigma_{r+k}
$$

Based on,

$$
\begin{aligned}
I_{0} & \equiv \sum_{\sigma_{r}= \pm 1}\left(1+\gamma \sigma_{r-1} \sigma_{r}\right)\left(1+\gamma \sigma_{r} \sigma_{r+1}\right) \sigma_{r} \\
& =2 \gamma\left(\sigma_{r-1}+\sigma_{r+1}\right) \\
I_{1} & \equiv \sum_{\sigma_{r+1}= \pm 1} I_{1}\left(1+\gamma \sigma_{r+1} \sigma_{r+2}\right) \\
& =2^{2} \gamma\left(\sigma_{r-1}+\gamma \sigma_{r+2}\right)
\end{aligned}
$$


we get,

$$
\begin{aligned}
I_{k} & \equiv \sum_{\sigma_{r+k}= \pm 1} I_{k-1}\left(1+\gamma \sigma_{r+k} \sigma_{r+k+1}\right) \sigma_{r+k} \\
& =2^{k+1} \gamma\left(\gamma^{k-1}+\gamma \sigma_{r-1} \sigma_{r+k+1}\right)
\end{aligned}
$$

$$
\begin{aligned}
\zeta_{k} & =\lim _{N \rightarrow \infty} \frac{1}{2^{N}} \sum_{\substack{\sigma_{1}= \pm 1, \cdots \sigma_{r-1}= \pm 1 \\
\sigma_{r+k+1}= \pm 1, \cdots \sigma_{N}= \pm 1}} I_{k} \prod_{i=1}^{r-2}\left(1+\gamma \sigma_{i} \sigma_{i+1}\right) \prod_{i=r+k+1}^{N-1}\left(1+\gamma \sigma_{i} \sigma_{i+1}\right) \\
& =\gamma^{k}
\end{aligned}
$$

From Eq. M4 and M9, we obtain,

$$
X_{l}=\mu^{2}\left[l+2 \sum_{k=1}^{l-1}(l-k) \gamma^{k}\right]
$$

Here, $\sum_{k=1}^{l-1}(l-k) \gamma^{k}$ is the well-known arithmetic-geometric series, and

$$
X_{l}=\mu^{2}\left\{2\left[\frac{l-\gamma^{l}}{1-\gamma}-\frac{\gamma\left(1-\gamma^{l-1}\right)}{(1-\gamma)^{2}}\right]-l\right\}
$$

Therefore,

$$
m_{s}^{l}=\frac{\mu}{l} \sqrt{2\left[\frac{l-\gamma^{l}}{1-\gamma}-\frac{\gamma\left(1-\gamma^{l-1}\right)}{(1-\gamma)^{2}}\right]-l}
$$

and obviously,

$$
m_{s}^{l}(T \rightarrow \infty)=\frac{\mu}{l^{1 / 2}}
$$

\section{Simulation of spin orientation states in 1D-IM}

The method to simulate the spin orientation states in 1D-IM by a computer is as the follows ${ }^{24}$ :

1. Simulate a spin chain of length $N$ with its $\sigma_{i}(i=1, \cdots N)$ initial state being completely disordered. The disorder initial state is constructed by generating a random number $r_{d}$, and if $r_{d}<0.5, \sigma_{i}=1$, otherwise $\sigma_{i}=-1$. Obviously, this corresponds to the case of $T \rightarrow$ $\infty$ of the spin chain.

2. The evolution of the spin chain from the initial state to thermal equilibrium one of a certain $T$ is determined by Glauber dynamics ${ }^{25}$, i.e. the transition probability from $\sigma_{i}$ to $-\sigma_{i}$ is,

$$
p_{i}=\frac{\exp \left[-\frac{J \sigma_{i}\left(\sigma_{i-1}+\sigma_{i+1}\right)}{k_{B} T}\right]}{\exp \left[\frac{J \sigma_{i}\left(\sigma_{i-1}+\sigma_{i+1}\right)}{k_{B} T}\right]+\exp \left[-\frac{J \sigma_{i}\left(\sigma_{i-1}+\sigma_{i+1}\right)}{k_{B} T}\right]}
$$

where $i=1, \cdots n$, and $\sigma_{0}=\sigma_{n+1}=0$.

The specific simulation process is as follows: (a) Generate a random number $r_{d}^{i}$ for any $i$ value, and if $r_{d}^{i}<p_{i}, \sigma_{i}=-\sigma_{i}$, otherwise $\sigma_{i}$ remains unchanged; (b) The above operation is performed once for each spin in the spin chain, which is called a Monte Carlo step; and (c) The Monte Carlo step is repeated $S$ times until the spin chain reaches thermal equilibrium.

3. The criterion parameter of thermal equilibrium selected in this paper is the average nearestneighbor anti-bond number $(d)$ in the spin chain,

$$
d \equiv \frac{1}{N-1} \sum_{i=1}^{N-1} \delta_{\sigma_{i},-\sigma_{i+1}}
$$

In which, $\delta_{\sigma_{i},-\sigma_{i+1}}$ is a special Kronecker $\delta$-function, i.e. for $\sigma_{i}=-\sigma_{i+1}, \delta_{\sigma_{i},-\sigma_{i+1}}=1$, otherwise $\delta_{\sigma_{i},-\sigma_{i+1}}=0$. This is because from $d$, the average nearest-neighbor positive- 
bond number in the spin chain is $1-d$, and then the average internal energy of each spin is $\frac{N-1}{N} J(2 d-1)$. Because 1D-IM is a canonical ensemble, and the thermal equilibrium condition of the ensemble is energy.

Fig. 5 (A, B and C) shows the modulated $d$ vs $S$ for series $T$ and $N$ in 1D-IM. It can be seen that: (a) When $S$ is large enough, $d$ tends to a stable value, indicating that the system is in thermal equilibrium; (b) With decreasing $T$, the greater $S$ is required for the system to reach the equilibrium; and (c) The larger the $N$, the smaller the fluctuation of $d$ when the system is at the equilibrium.

For comparison, this paper also simulates $d$ vs $S$ for series $T$ and $N$ of 1D-IM whose initial state is completely ordered $(d=0)$, as shown in Fig. $5(\mathrm{D}, \mathrm{E}$ and F). The results show that when $S$ is large enough, $d$ tends to the value of the initial state of complete disorder, indicating that the spin chain is indeed in thermal equilibrium.
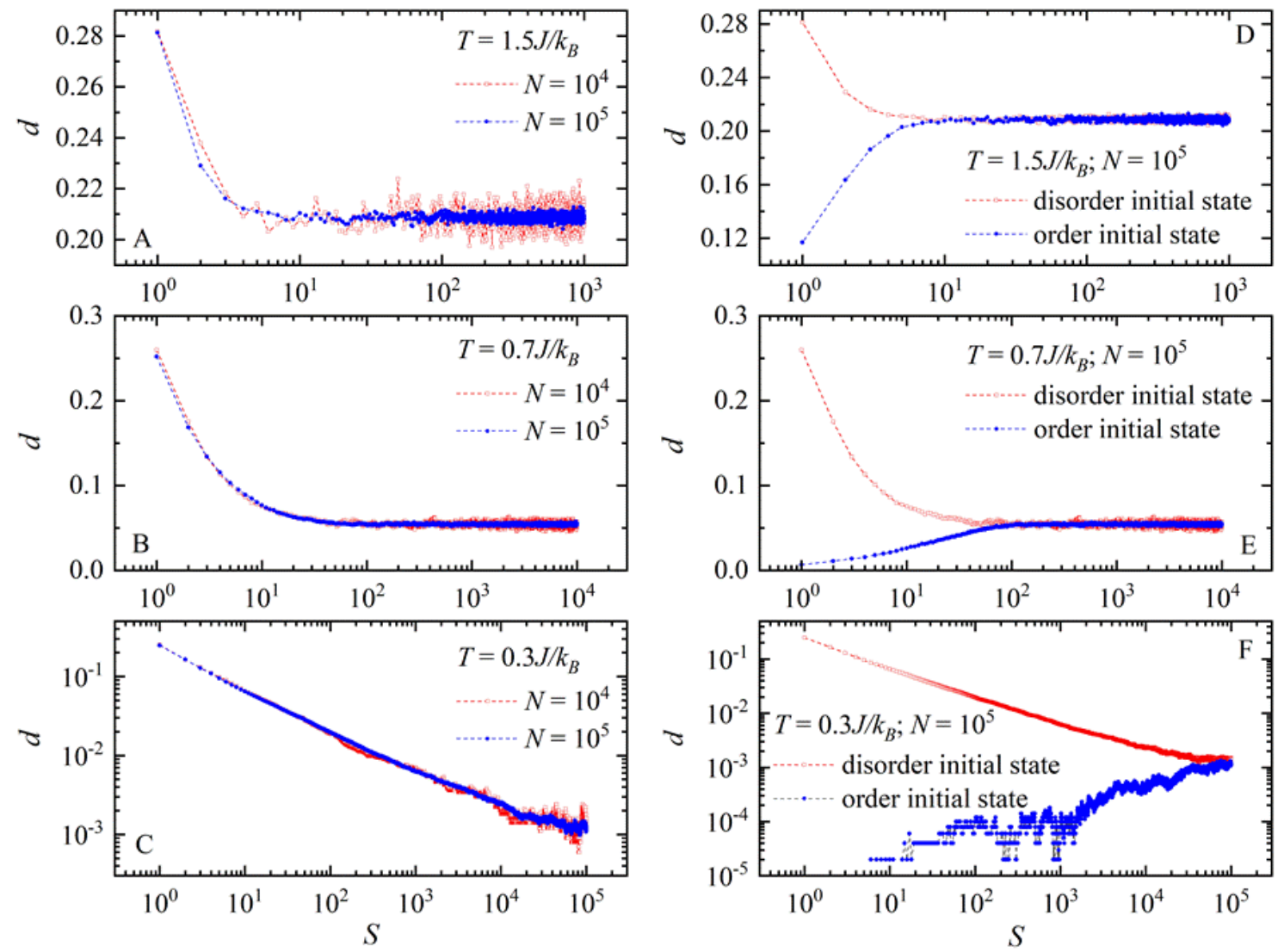

Fig. 5 Modulated average anti-bond number $(d)$ vs Monte Carlo steps $(S)$ for series temperature $(T)$ and spin number $(N)$ with the disorder initial spin state $(\mathrm{A}, \mathrm{B}$, and $\mathrm{C})$ of one-dimensional Ising model. For series $T, d$ vs $S$ of the disorder and order initial spin states in the model when $N=10^{5}$ (D, E, and F).

\section{DATA AVAILABILITY}

The data that support the plots and other findings of this paper are available from the corresponding author upon a reasonable request.

\section{CODE AVAILABILITY}

The code is available from the corresponding author upon a reasonable request.

\section{REFERENCES}


1. Haggkvist, R. et al. On the Ising model for the simple cubic lattice. Adv. Phys. 56, 653-755 (2007).

2. Ising, E. Report on the theory of ferromagnetism. Z. Phys. 31, 253-258 (1925).

3. McCoy, B. M. \& Wu, T. T. The two-dimensional Ising model. (Dover Publications, Inc., Mineola, New York, 2014).

4. Young, J. T., Gorshkov, A. V., Foss-Feig, M. \& Maghrebi, M. F. Nonequilibrium Fixed Points of Coupled Ising Models. Phys. Rev. X. 10, (2020).

5. Christiansen, H., Majumder, S., Henkel, M. \& Janke, W. Aging in the Long-Range Ising Model. Phys. Rev. Lett. 125, (2020).

6. Walker, N., Tam, K. \& Jarrell, M. Deep learning on the 2-dimensional Ising model to extract the crossover region with a variational autoencoder. Sci. Rep. 10, (2020).

7. Wang, B., Hu, F., Yao, H. \& Wang, C. Prime factorization algorithm based on parameter optimization of Ising model. Sci. Rep. 10, (2020).

8. Imanaka, Y., Anazawa, T., Kumasaka, F. \& Jippo, H. Optimization of the composition in a composite material for microelectronics application using the Ising model. Sci, Rep. 11, (2021).

9. Weiss, P. Molecular field and ferro-magnetism. Phys. Z. 9, 358-367 (1908).

10. Chikazumi, S. Physics of ferromagnetism. (Oxford University Press, Oxford, 2009).

11. Moriya, Y., Kawaji, H., Tojo, T. \& Atake, T. Specific-heat anomaly caused by ferroelectric nanoregions in $\mathrm{Pb}\left(\mathrm{Mg}_{1 / 3} \mathrm{Nb}_{2 / 3}\right) \mathrm{O}_{3}$ and $\mathrm{Pb}\left(\mathrm{Mg}_{1 / 3} \mathrm{Ta}_{2 / 3}\right) \mathrm{O}_{3}$ relaxors. Phys. Rev. Lett. 90, 205901 (2003).

12. Kleemann, W., Dec, J., Shvartsman, V. V., Kutnjak, Z. \& Braun, T. Two-dimensional Ising model criticality in a three-dimensional uniaxial relaxor ferroelectric with frozen polar nanoregions. Phys. Rev. Lett. 97, 65702 (2006).

13. Tachibana, M., Sasame, K., Kawaji, H., Atake, T. \& Takayama-Muromachi, E. Thermal signatures of nanoscale inhomogeneities and ferroelectric order in $\left[\mathrm{PbZn}_{1 / 3} \mathrm{Nb}_{2 / 3} \mathrm{O}_{3}\right]_{1-\mathrm{x}}\left[\mathrm{PbTiO}_{3}\right]_{\mathrm{x}}$. Phys. Rev. B. 80, 94115 (2009).

14. Granzow, T., Woike, T., Wohlecke, M., Imlau, M. \& Kleemann, W. Change from 3D-Ising to random field-Ising-model criticality in a uniaxial relaxor ferroelectric. Phys. Rev. Lett. 92, 65701 (2004).

15. Stock, C. et al. Interplay between static and dynamic polar correlations in relaxor $\mathrm{Pb}\left(\mathrm{Mg}_{1 / 3} \mathrm{Nb}_{2 / 3}\right) \mathrm{O}_{3}$. Phys. Rev. B. 81, 144127 (2010).

16. Gehring, P. M. et al. Reassessment of the Burns temperature and its relationship to the diffuse scattering, lattice dynamics, and thermal expansion in relaxor $\mathrm{Pb}\left(\mathrm{Mg}_{1 / 3} \mathrm{Nb}_{2 / 3}\right) \mathrm{O}_{3}$. Phys. Rev. B. 79, 224109 (2009).

17. Cross, L. E. Relaxor ferroelectrics. Ferroelectrics. 76, 241-267 (1987).

18. Shvartsman, V. V. \& Lupascu, D. C. Lead-free relaxor ferroelectrics. J. Am. Cera. Soc. 95, 1-26 (2012).

19. Shvartsman, V. V., Dkhil, B. \& Kholkin, A. L. Mesoscale domains and nature of the relaxor state by piezoresponse force microscopy. Annu. Rev. Mater. Res. 43, 423-449 (2013).

20. Zhang, L. L. \& Huang, Y. N. Theory of relaxor-ferroelectricity. Sci. Rep. 10, 50601 (2020).

21. Kittel, C. Physical theory of ferromagnetic domains. Rev. Mod. Phys. 21, 541-583 (1949).

22. Nakatani, N. Observation of Ferroelectric Domain Structure in TGS. Ferroelectrics. 413, 238-265 (2011).

23. Potnis, P. R., Tsou, N. \& Huber, J. E. A Review of Domain Modelling and Domain Imaging Techniques in Ferroelectric Crystals. Materials. 4, 417-447 (2011).

24. Fulco, U. L., Nobre, F. D., Da Silva, L. R. \& Lucena, L. S. Investigation of critical properties in the twodimensional site-diluted Ising ferromagnet. Physica a. 297, 131-141 (2001).

25. Glauber, R. J. Time-dependent statistics of Ising model. J. Math. Phys. 4, 294 (1963).

26. Kimura, T., Tomioka, Y., Kumai, R., Okimoto, Y. \& Tokura, Y. Diffuse phase transition and phase separation in Cr-doped $\mathrm{Nd}_{1 / 2} \mathrm{Ca}_{1 / 2} \mathrm{MnO}_{3}$ : A relaxor ferromagnet. Phys. Rev. Lett. 83, 3940-3943 (1999).

27. Belik, A. A., Yokosawa, T., Kimoto, K., Matsui, Y. \& Takayama-Muromachi, E. High-pressure synthesis and properties of solid solutions between $\mathrm{BiMnO}_{3}$ and $\mathrm{BiScO}_{3}$. Chem. Mat. 19, 1679-1689 (2007).

28. Chattopadhyay, S., Ayyub, P., Palkar, V. R. \& Multani, M. Size-induced diffuse phase-transition in the nanocrystalline ferroelectric $\mathrm{PbTiO}_{3}$. Phys. Rev. B. 52, 13177-13183 (1995).

29. Muthuselvam, I. P. \& Bhowmik, R. N. Grain size dependent magnetization, electrical resistivity and magnetoresistance in mechanically milled $\mathrm{La}_{0.67} \mathrm{Sr}_{0.33} \mathrm{MnO}_{3}$. J. Alloys Comp. 511, 22-30 (2012).

30. Landau, L. Zur Theorie der Phasenumwandlungen II. Phys. Z. Soviet Union. 545, 26-35 (1937).

31. Ehrenfest, P. Phase conversions in a general and enhanced sense, classified according to the specific singularities of the thermodynamic potential. Proc. Konin. Aka. .Weten. Amst. 36, 153-157 (1933).

32. Onsager, L. Crystal statistics I: A two-dimensional model with an order-disorder transition. Phys. Rev. 65, 117-149 (1944).

33. Yang, C. N. The spontaneous magnetization of a 2-dimensional Ising model. Phys. Rev. 85, 808-815 (1952).

34. de Souza, S. M. \& Rojas, O. Quasi-phases and pseudo-transitions in one-dimensional models with nearest neighbor interactions. Sol. Stat. Comm. 269, 131-134 (2018). 
35. Rojas, O. A Conjecture on the Relationship Between Critical Residual Entropy and Finite Temperature Pseudo-transitions of One-dimensional Models. Bra. J. Phys. 50, 675-686 (2020).

36. Rojas, O., Strecka, J., Derzhko, O. \& de Souza, S. M. Peculiarities in pseudo-transitions of a mixed spin$(1 / 2,1)$ Ising-Heisenberg double-tetrahedral chain in an external magnetic field. J. Phys. - Cond. Mat. 32, (2020).

37. Huang, Y. N., Wang, Y. N. \& Shen, H. M. Internal-friction and dielectric loss related to domain-walls. Phys. Rev. B. 46, 3290-3295 (1992).

38. Huang, Y. N. et al. Domain freezing in potassium dihydrogen phosphate, triglycine sulfate, and CuAlZnNi. Phys. Rev. B. 55, 16159-16167 (1997).

39. Deblois, R. W. Domain wall motion in metals. J. Appl. Phys. 29, 459-467 (1958).

40. Deleeuw, F. H., Vandendoel, R. \& Enz, U. Dynamic properties of magnetic domain-walls and magneticbubbles. Rep. Prog. Phys. 43, 689 (1980).

41. Kaufman, B. Crystal statistics. 2. Partition function evaluated by spinor analysis. Phys. Rev. 76, 12321243 (1949).

42. Ferdinand, A. E. \& Fisher, M. E. Bounded and inhomogeneous Ising models: I. specific-heat anomaly of a finite lattice. Phys. Rev. 185, 832-846 (1969).

43. Brendel, K., Barkema, G. T. \& van Beijeren, H. Nucleation times in the two-dimensional Ising model. Phys. Rev. E. 71, (2005).

44. Lin, Y. \& Wang, F. Linear relaxation in large two-dimensional Ising models. Phys. Rev. E. 93, (2016).

45. Kumar, A. et al. Atomic-resolution electron microscopy of nanoscale local structure in lead-based relaxor ferroelectrics. Nat. Mat. 20, (2021).

46. $\mathrm{Li}$, F. et al. Giant piezoelectricity of $\mathrm{Sm}$-doped $\mathrm{Pb}\left(\mathrm{Mg}_{1 / 3} \mathrm{Nb}_{2 / 3}\right) \mathrm{O}_{3}-\mathrm{PbTiO}_{3}$ single crystals. Science. 364, 264 (2019).

47. Krogstad, M. J. et al. The relation of local order to material properties in relaxor ferroelectrics. Nat. Mater. 17, 718-724 (2018).

48. Li, J. et al. Scale-invariant magnetic textures in the strongly correlated oxide NdNiO3. Nat. Comm. 10, (2019).

49. Kim, Y., Yuk, H., Zhao, R., Chester, S. A. \& Zhao, X. Printing ferromagnetic domains for untethered fast-transforming soft materials. Nature. 558, 274 (2018).

50. Scott, J. F. Applications of modern ferroelectrics. Science. 315, 954-959 (2007).

\section{ACKNOWLEDGMENTS}

We sincerely thank Prof. W. Kleemann, M. E. Manley and J. Petzelt for their enlightening discussions on the mechanism of diffuse phase-transition. This work is supported by the Open Project of Xinjiang Key Laboratory (Grant No. 2021D04015) and Xinjiang Tianshan Youth Program (Grant No. 2017Q038).

\section{AUTHOR CONTRIBUTIONS}

Y-N.H. and L-L.Z. conceived this article together. Y-N.H. calculated the all-size spontaneousmagnetization. L.Z. did the numerical calculations and made plots.

\section{COMPETING INTERESTS}

Authors declare that they have no competing interests.

\section{ADDITIONAL INFORMATION}

All data are available in the main text.

Correspondence and requests for materials should be addressed to Y.-N.H. 